\title{
Eine neue Deutung der großen Initiale I des 7. Buches der Vesalschen «Fabrica»*
}

\author{
mit 3 Abbildungen \\ Aus dem Anatomischen Institut Fribourg (Schweiz) \\ (Direktor: Prof.Dr.med.A.FALLER) \\ Von Adolf Faller

\section{Einleitung}

In seinem Système de politique positive (1851-54) sagt Auguste Comte: "Aucune science ne saurait être comprise sans sa propre histoire, toujours inséparable de l'histoire générale de l'humanité». Wer eine medizingeschichtliche Einzelheit deuten will, darf dies nur aus dem Geist der damaligen Zeit tun. In diesem Sinn möchte ich eine neue Deutung der großen Initiale I am Anfang des 7. Buches von Vesals «Fabrica» versuchen.

Um die Mitte des 16. Jahrhunderts hatte der Buchdruck in Basel einen ungeahnten Aufschwung genommen. Künstlerisch wertvolle Titelblätter und Illustrationen schmücken die meisten großen Ausgaben dieser Zeit.

Die erste Auflage der «Fabrica» erschien 1543 bei Hans Herbster, welcher nach der Sitte seiner Zeit seinen Namen in Johannes Oporinus abgeändert hatte. Ihr folgte schon 1555 eine zweite Auflage, welche als die schönste Vesal-Ausgabe gilt. Die Papierqualität ist besser und die Drucktypen sind größer. Es mag das wohl der Grund dafür gewesen sein, daß die kleinen Anfangsbuchstaben neu geschnitten werden mußten. Sie sind in der zweiten Auflage sieben Zeilen hoch.

\section{Buchschmuck durch Initialen und dekorative Verwendung der Kindergestalt}

In bewußter Nachahmung der alten Manuskripte wurden die Buch- und die Kapitelanfänge mit verzierten Initialbuchstaben geschmückt. Nach Anson (1949) stammen die ersten gedruckten Initialen aus dem Jahre 1457. In den ersten deutschen Bilderbibeln gegen Ende des 15. Jahrhunderts wurde versucht, den schmückenden Initialen den Charakter von Textabbildungen zu geben. Man schrieb den Anfangsbuchstaben in eine Szene

* Dem einstigen Privatdozenten Dr.med.et Dr.phil. G. Wolf-Heidegger zum 60. Geburtstag in steter Dankbarkeit von seinem ersten Doktoranden und jetzigen Kollegen gewidmet. 
ein, welche sich auf eine bestimmte Stelle des Textes bezieht. Mit Hans Holbeiv dem Jüngeren kommen die Initial-Alphabete auf. Wir kennen von ihm ein Bauern-Alphabet (1522), ein Totentanz-Alphabet (1524) und ein Kinder-Alphabet (1525). Die für CuRio in Basel entworfenen Kinderszenen erfreuten sich bald großer Beliebtheit. Nackte Kinder, mit oder ohne Flügel, als Dekorationselement kennt man schon aus der römischen Kaiserzeit: Putti finden sich auf den Wandmalereien von Pompeji. Sie dienen häufig auch als Brunnenfiguren. Die Kunst der Renaissance übernahm diese nackten Kinder. Als Putti feierten sie ihren großen Triumph im Barock und im Rokoko. Die Basler Vesal-Ausgabe läßt sie in anatomischen oder klinischen Szenen auftreten. Diese spielerische Darstellung des Anatomen und Chirurgen, der täglich mit Krankheit, Zerfall und Tod zu tun hat, läßt humorvoll über die düstere Seite des Lebens hinwegsehen.

Auch die kirchliche Kunst verwendete gelegentlich die Kindesgestalt. Die Passion des «Schmerzenskindes» ist in der Graphik des 15. Jahrhunderts nicht selten. Auch für Auferstehungsbilder bediente man sich des Jesuskindes, um die Einheit von Geburt und Wiedergeburt zu betonen. Der neugeborene und der wiedergeborene Christus sind derselbe. Der Auferstandene tritt zum zweitenmal in die Welt. Am bekanntesten ist wohl die Darstellung der Auferstehung in Kindesgestalt durch Lukas Cranach den. Älteren. Diese uns merkwürdig anmutende Darstellungsart ist nicht ohne Bedeutung für die Erklärung der Initiale I.

\section{Die kleinen Initialen der «Fabrica»}

VESAL hatte einen guten Blick für die künstlerische Wirkung eines Holzschnittes. Er gestaltete seine Sektionsbefunde in «frühbarocker Denkweise » (Pfister, 1942). Man denke etwa an den Bauchsitus des 5. Buches, welcher einem antiken Torso eingezeichnet ist. HolläNDER (1903) hat die großen und die kleinen Initialen dem Holzschneider der anatomischen Tafeln JAN Stephan van Calcar zugeschrieben: "Van Calcar hat zur Verzierung einer großen Reihe von Initialen medizinische Vorgänge benützt, und gerade an der derben Zugabe erkennen wir den Bauernhumor seines Landes ... Ein Italiener konnte obszön sein, wie manchmal GARACCI, aber solchen derben Humors war nur ein Verwandter von Teniers und Breughel fähig.» Dieser Ansicht schließt sich Anson (1949) an: "the blocks and especially those of the larger series are believed to have been done by CALGAR, who also designed the anatomical plates." Für die kleinen Initialen der ersten 
Auflage erscheint es wenig wahrscheinlich: die Technik ist grob, die Schraffur des Hintergrundes wenig regelmäßig, die Einzelheiten sind wenig sorgfältig behandelt. Da Calcar 1546 starb, ist es ziemlich sicher, daß die kleinen Initialen der 2. Auflage von 1555 nicht von ihm geschnitten wurden. Herrlinger (1950) hält es für wenig wahrscheinlich, «daß sich der Verfasser des anatomischen Textes und sein Zeichner - oder auch nur der Letztere - mit Arbeiten belastet haben, die herkömmlicherweise Sache des Verlegers sind.» Er wirft die Frage auf, ob nicht OpoRINus «die Initialen hat schneiden lassen oder gar selbst geschnitten hat». Es ist interessant, daß die ebenfalls in Basel gedruckte Praxeos Medicinae (1625) von Felix PlatTER die Vorreden der drei Bücher mit den Initialen C, S und A der «Fabrica» beginnt.

Sechs Anfangsbuchstaben klinischen Inhaltes: Chirurgie E, F, H, V; Urologie A und Geburtshilfe I, stehen elf Initialen mit Szenen aus der Anatomie gegenüber: Vergleichende Anatomie M, Q, R, S, T; Leichenbeschaffung L, N, O; Skelettierung C, P; Schädelsektion D. Eine besondere Stellung nimmt die kleine Initiale $\mathrm{L}$ des Kapitels 51 des zweiten Buches ein, welches «de Recti intestini musculis» handelt und mit dem Satz beginnt: «Laudatur numquam satis natura.» Der Mittelstrich, den die anderen Buchstaben nicht haben, gibt ihr Reliefcharakter. Das humoristische Bildchen wird von zwei dicken und einer dünnen Linie umrandet. Herrlinger (1963) hat darauf hingewiesen, daß es sich um eine Kopie aus dem Kinder-Alphabet von HoLbers handelt. Die Deutung der dargestellten Szenen ist nicht immer einfach. Zahlreiche Autoren haben sich daran versucht: Rотн 1892, HoLlaender 1903, Spencer 1923, Chaigneau 1935, Metzger 1935, RosenKranz 1937, Schmutzer 1938, Francis 1943, Monteiro 1943 und 1952, Anson 1945 und 1949, Faller 1948, 1951 und 1952, Herrlinger 1950, 1953 und 1963, Lambert 1952, Ollenshaw 1952, Van Driessche 1954, Wells 1962, Wolf-Heidegger und Cetto 1966.

Ein bisher nicht berücksichtigter Gesichtspunkt sind die Beziehungen mancher Initialen zum Neuen Testament. Bei der kleinen Initiale $\mathbf{O}$ haben Bilder der Decollatio des Täufers als Anregung gedient. Die Komposition der kleinen Initiale L erinnert an eine Kreuzabnahme. Der Künstler war so stark von seinem Vorbild beeinflußt, daß er in der Puttiszene Joseph von Arimathäa als Erwachsenen darstellte, was zuerst HerrLinger aufgefallen war (1963). Die beiden Hälften der großen Initiale I deuten wir als Grablegung und Auferstehung (FALLER 1951). Wir werden dieser Frage ein eigenes Kapitel widmen. 
Die Szenen der kleinen Initialen O und L sind von Darstellungen christlicher Ikonographie beeinflußt. Die völlige Umwandlung in eine Kinderszene ist unterblieben: In $\mathrm{O}$ finden wir einen einzigen Putto, in $\mathrm{L}$ nur drei Putti unter lauter Erwachsenen.

Abb. 1

Der berittene Führer der geharnischten Soldaten und der Henker tragen eine turbanartige orientalische Kopf bedeckung, wohl in Erinnerung an eine Darstellung der Decollatio des Täufers. Das eigentliche Abschneiden des Kopfes läßt sich schon auf romanischen Säulenkapitellen nachweisen

Abb. 2

Die Szene erinnert an eine Kreuzabnahme. Der Künstler war so stark von seinem Vorbild beeinflußt, daß er es unterlassen hat, den Mann auf der Leiter, der wohl Joseph von Arimathäa darstellt, in einen Putto umzuwandeln

Der Zusammenhang der großen Initialen mit dem anatomischen Text
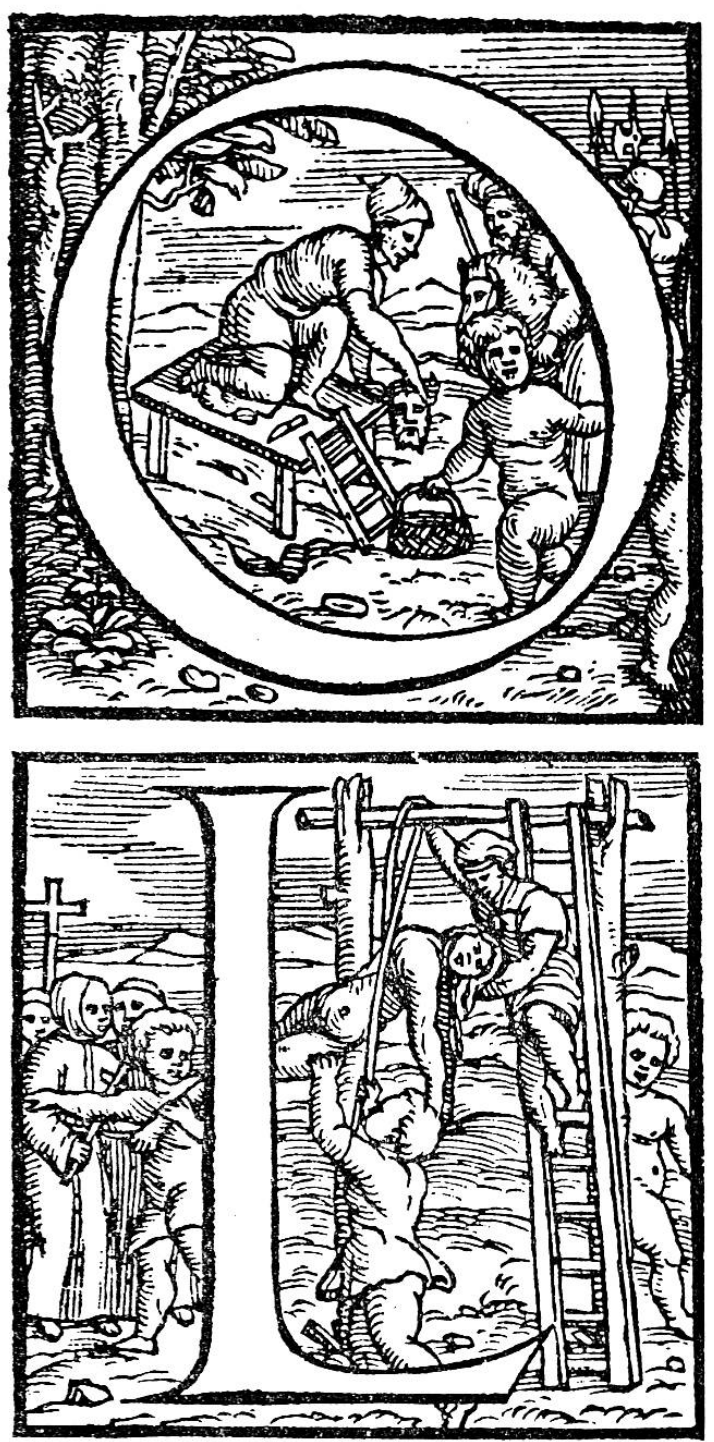

Die großen Initialen der Dedication an KaRL v. und der sieben Bücher sind in der ersten und der zweiten Auflage die gleichen. Es handelt sich um I, O, Q, T und V. Die Blöcke sind zwölf Zeilen hoch. Ihre Breite schwankt zwischen 71 und $75 \mathrm{~mm}$, ihre Höhe zwischen 72 und $75 \mathrm{~mm}$. Der Vermessung der Initialen unter Angabe ihres Vorkommens hat Loetzke (1970) eine besondere Arbeit gewidmet. Die Unregelmäßigkeiten der Blöcke lassen vermuten, daß nicht alle Druckstöcke gleichzeitig geschnitten wurden. Sobald ein Buch druckfertig vorlag, wurde eine dazu passende Initiale angefertigt. 
Die Initiale V leitet die Dedicatio und das 5. Buch ein. Der Buchstabe O steht am Anfang des 1. Buches und wiederholt sich zu Beginn des 3. Buches. Das 2. Buch weist als einziges zwei große Initialen auf: T und Q. Mit 227 Folioseiten und 62 Kapiteln ist es das umfangreichste. Mit T beginnt ebenfalls das 4.Buch. Q wiederholt sich am Anfang des 6. Buches. Beim 7.Buch wird erstmals der Buchstabe I gebraucht. Wo eine große Initiale zum erstenmal verwendet wird, läßt sich stets ein sinnvoller Zusammenhang mit dem anatomischen Text nachweisen. Bei den Repetitionen ist das nur ausnahmsweise der Fall.

Die große Initiale V ist mythologisch-allegorischen Inhaltes, was bei einer Widmung aus der ausgehenden Renaissance kaum erstaunen wird, wählte sie ihre Allegorien doch größtenteils aus der klassischen Mythologie. Die Allegorie drückt einen abstrakten Sachverhalt in einer konkreten Gestalt aus. Damit haftet ihr im Gegensatz zum Symbol, das objektiv Gültiges aussagt, etwas Willkürliches an, was unserer sachbezogenen Denkart oft schwer einfühlbar ist. Das Tryptichon vom musikalischen Wettstreit zwischen Apoll und dem phrygischen Silen Marsyas läßt Deutungen auf verschiedenen Ebenen zu :

Der Wettstreit der Sterblichen mit den Göttern ist ein gewagtes Unterfangen: Der Anatom, welcher die Geheimnisse des Menschenleibes ergründet, dringt in die verlassene Werkstatt des Schöpfers ein. Die Sektion eines menschlichen Körpers, besonders aber eines Kopfes war damals noch ein gewagtes Unterfangen. Ein gewagtes Unterfangen war es aber auch, gegen die unbeschränkte Autorität eines GaLEnos aufzutreten und ihn derAffen-Anatomie zu beschuldigen.

Der Töter Apoll ist auch Heil-Gott: In der Ilias versendet er mit silbernem Bogen die tödlichen Pfeile. So stellt ihn das Bildwerk des Belvedere dar. Der Sohn von Apoll ist der Heilgott Asklepios. Dieser Doppelcharakter Apolls weist auf den Doppelcharakter der Medizin hin, welche heilt oder tötet.

Abgezogene Menschenhaut vergegenwärtigt die anatomische Sektion: Der den Marsyas schindende Apoll verkörpert den Anatomen. Man kannte ja damals die Pinzette noch nicht, sondern verwendete beim Präparieren den einfachen oder den doppelten Hacken (Faller, 1948). Das Abziehen von

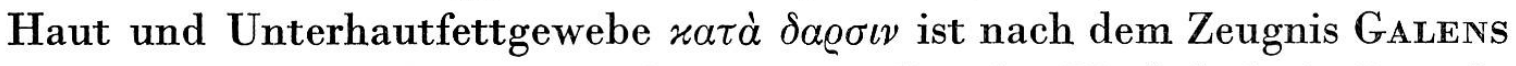
das Vorgehen des Herophilos beim Darstellen des Muskelreliefs. Der abgezogenen und gegerbten Menschenhaut wurden besondere Kräfte zugeschrieben. Auf einer Abbildung des anatomischen Theaters Leyden von 1610 läßt sich eine elegant gekleidete Dame eine abgezogene Menschenhaut demonstrieren. Die Description of the Body of Man von Helkiah Crooke 
(London 1631), ein Sammelwerk aus Bauhin und Laurentius, zeigt auf dem Titelblatt einen mit Messer bewaffneten Muskelmann, der in seiner Rechten die eigene abgezogene Haut emporhält. Eine Menschenhaut trägt den Titel der Anatomia reformata (Haag 1655) des jüngeren Bartholin. Auf dem Titelblatt des Clavis medicinae Johannis Muralti (Zürich 1677) halten fliegende Putti eine abgezogene Haut mit der Titelinschrift. Muralts Anatomisches Collegium gehalten zu Zürich im Jahre Christi 1868 (Nürnberg 1687) zeigt wiederum Menschenhaut, die aber hier von einem Knochenmann dem Publikum entgegengehalten wird. In den Tabulae anatomicae von J.A.Kulmus (Danzig 1725) hängt die abgezogene Haut mit drauf geschriebenem Titel an der Wand.

Das 1. Buch beginnt mit der Majuskel O. Die von Vesal geübte Kochmazeration der Knochen und Knorpel, die im 40. Kapitel eingehend beschrieben wird, bedeutete gegenüber der traditionellen Mazeration in durchlöcherter Kiste, welche ins fließende Wasser gehängt wurde, einen erheblichen Fortschritt. Die alte Methode, welche Vesalius als «molesta, sordida ac difficilis» kennzeichnet, ist in der kleinen Initiale C dargestellt (Faller 1948). Kochmazeration wurde schon in den Kreuzzügen des 11. und 12. Jahrhunderts geübt, um die Gebeine gefallener Ritter in die Heimat mitnehmen zu können. Berengar brauchte laut Commentaria in Mundinum (Bologna 1521) die Kochmazeration zur Darstellung der Fußwurzelknochen. Auch Nicolao Massa kannte die «Coctio», wie sein Liber introductorius anatomiae sive dissectionis corporis humani (Venedig 1536) beweist. Beim großen Buchstaben $O$ ist der Zusammenhang mit dem Inhalt des 1. Buches eindeutig klar.

Das durch seine Muskelmänner berühmte 2. Buch weist mit beiden Initialen auf die Tieranatomie hin. Als Ersatz für die nur schwierig zu beschaffenden menschlichen Leichen wurden häufig Hunde, wie in T, und Schweine wie es Q zeigt, verwendet. Zugleich bringen die zwei Buchstaben die beiden damals geübten anatomischen Methoden zur Darstellung: Kadaveranatomie und Vivisektion. Beide Methoden erwähnt VeSAL ausdrücklich im 2. Buch: «Quidam enim dicebant anatomiam in moruis animalibus debere fieri. Alii vero consideratione satis provida asserebant animalia viva ad anatomizandum esse potiora ...» Der Querbalken des T hat beim Künstler die Ideenassoziation von Galgen, Hängen und Erdrosseln geweckt. Wichtiger ist die Vivisektion im Médaillon des Buchstabens Q. Solche Experimente machte bereits GALEN an Schweinen und Ziegen, um die Tätigkeit der Atemmuskeln zu untersuchen. GaLen kannte bereits die Wirkung der Er- 
öffnung der Pleurahöhlen und der Schädigung der Zwerchfellnerven. Im 2. Buch behandelt VesaL die Wirkung der Interkostalmuskeln und des Zwerchfells. Die Initiale Q mit ihrer Vivisektionsszene ist aber auch im 6.Buch am richtigen Platz, welches «cordi ipsique famulantibus organis» gewidmet ist. Wenn einer der Putti aus einem Kompendium vorliest, so könnte es sich sehr wohl um die Salernitaner Schweine-Anatomie Demonstratio corporis animalis des MaURus handeln. T und $Q$ zeigen spitz zulaufende Seziermesser. Vesal zog die deutschen und französischen Rasiermesser, "quibus tonsores in abradandis pilis utuntur», den gerade geschliffenen englischen und italienischen Schermessern vor. Mit spitz zulaufenden Messern läßt sich noch auf engstem Raum genau präparieren. Die gerade geschliffenen Messer eignen sich viel weniger dazu. Der sinnvolle Zusammenhang mit dem Text des 2. Buches ist eindeutig gegeben. Was für die Dedikation, das 1. und das 2. Buch, Gültigkeit hat, dürfte auch für die große Initiale des 7.Buches erwartet werden. Die Majuskel I erscheint ja nur an dieser Stelle.

\section{Die Deutung der großen Initiale I}

Das 7.Buch der «Fabrica» behandelt auf siebzig Folioseiten das Gehirn, die Hirnhäute und die Blutversorgung beider. Der Sinnzusammenhang der Majuskel mit dem Inhalt des 7. Buches ist keineswegs in die Augen springend.

Anson (1949) meint: "Cherubic curators secure an anatomical specimen in letter I by robbing a grave; here, outdoors and against a background of masonry arches, the putti exhume a corpse - one holding a candle to illuminate the crypte, another keeping watch. Couriously, a soldier, present a seeming sympathetic, does not interfere with the scientific garnering of laboratory material." Dieser Deutung folgten Metzger (1953), Lambert (1952) und van Driessche (1954). Auch Herrlinger (1963) nimmt «nächtlichen Leichenraub» an und fügt hinzu: «Die Handlung ist auf die linke Hälfte beschränkt. Die Bedeutung der drei Putten der rechten Hälfte bleibt zum Teil unklar, insbesondere die Fahne.» Auch Wolf-Heidegger und Cетто (1966) sprechen vom «Ausgraben eines Leichnams zu Sektionszwecken durch Putten». Damit wäre eine nur vage angedeutete Beziehung zum Inhalt des 7. Buches gegeben. Eine andere, viel enger an den Inhalt des 7. Buches anschließende Deutung scheint mir möglich: Das Gehirn, als Zentralorgan des Lebens, hat den Holzschneider an Leib und Seele, an Begräbnis und Auferstehung denken lassen. Der Balken des I unterteilt die Initiale in zwei voneinander unabhängige Hälften, deren Figurengrup- 
pen links die Form eines $\mathrm{V}$ und rechts diejenige eines umgekehrten $\mathrm{V}$ zeigen. Im einen Feld bildet das Haupt des zu Begrabenden, im anderen Feld die Fahne den Schwerpunkt. In ähnlicher Weise zeigt ja auch die Initiale V drei Szenen, die sich in den drei durch die Balken des V herausgeschnittenen Dreiecken abspielen.

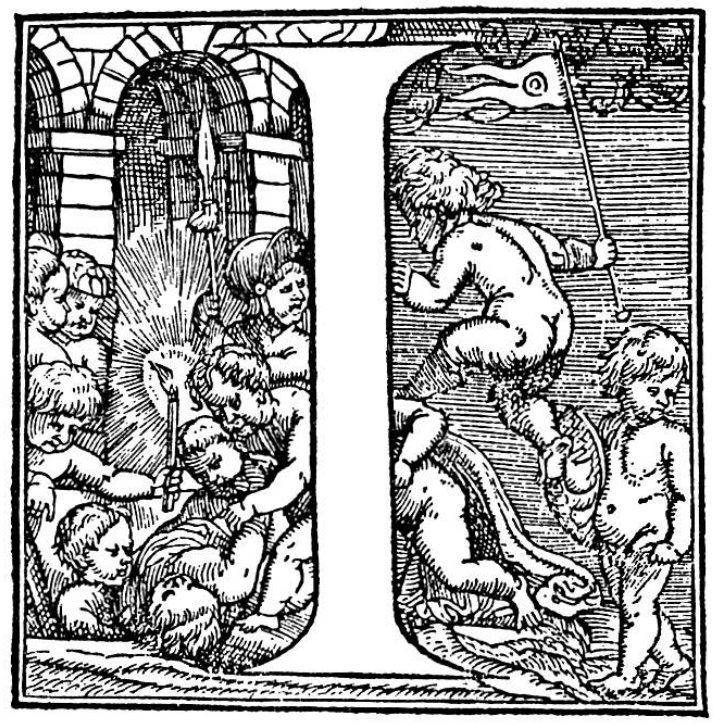
Abb. 3
Die große Initiale zu Anfang des 7. Buches der «Fabrica » zeigt zwei voneinander un- abhängige Bildhälften. Die Komposition der linken Szene hat die Gestalt eines V, diejenige der rechten die Gestalt eines um- gekehrten V. Links ist die Grablegung, rechts die Auferstehung dargestellt. Die Verwendung von Putti gibt dem Ganzen etwas Spielerisches. Die Verweltlichung der religiösen Darstellung wird zur Alle- gorie von Tod und ewigem Leben

Begräbnis und Auferstehung Christi sind dem christlichen Glauben des 16. Jahrhunderts gewohnte Symbole für leiblichen Tod und ewiges Leben. Die linke Hälfte der Initiale I stellt 8 Putti dar. In der Mitte der Gruppe leuchtet das Kerzenlicht oder die Fackel. Es handelt sich um ein Begräbnis bei Nacht und unter militärischer Bewachung. Das Grab ist ein durch eine Platte verschlossenes Gewölbe. Die Gestalt des Wächters mit Helm und Lanze macht die Deutung als Leichenraub unwahrscheinlich, während sie zu einer Grablegung durchaus paßt; die Hohenpriester und Pharisäer hatten sich nach Matthäus 27/63-66 bei Pilatus eine Bewachung des Grabes erbeten: „Herr, wir erinnern uns, daß jener Betrüger bei seinen Lebzeiten gesagt hat: Nach drei Tagen werde ich auferstehen. Laß darum das Grab bis zum dritten Tag bewachen; sonst könnten die Jünger kommen, ihn stehlen und dann zum Volke sagen: Er ist von den Toten auferstanden. Dann wäre der letzte Betrug noch schlimmer als der erste.' Pilatus erwiderte ihnen : ,Ihr sollt eine Wache haben; gehet hin und bewachet das Grab, wie es euch gut dünkt. 'Sie gingen hin, sicherten das Grab mit der Wache und versiegelten den Stein. Der Soldat erklärt sich somit als Kontrollorgan. Der Putto, welcher die Fackel hält, stützt die auf die Kante gestellte Grabplatte. Sein Kamerad ist bereits einige Stufen in das Grabgewölbe hinuntergestiegen 
und nimmt den Leichnam von zwei anderen Putti entgegen, die uns die Gestalt des Wächters zum Teil verdecken. Der rechte Arm der Leiche liegt noch auf der linken Schulter des einen Trägers. Der zweite verlangsamt die Bewegung des Hinlegens. Vor dem steinernen Pfeiler betrachten zwei andere Putti, von denen der eine eine Art Mütze trägt, ernsthaft diese Szene. In ihnen dürfen wir nach Johannes 19/38-39 Joseph von Arimathäa und Nicodemus sehen: «Joseph von Arimathäa, der ein Jünger Jesu war, aber aus Furcht vor den Juden nur im geheimen, erbat sich darauf von Pilatus die Erlaubnis, den Leichnam Jesu abzunehmen. Pilatus gestattete es. Er ging also hin und nahm den Leichnam ab. Auch Nicodemus, der einst des Nachts zu ihm gekommen war, fand sich ein und brachte eine Mischung von Myrrhe und Aloes, wohl an hundert Pfund.»

Die rechte Seite der Initiale zeigt nur drei Figuren. Unten sind die grasbewachsenen Schollen der Erde angedeutet. Auf ihr stehen zwei Putti. Den einen, der einen reich verzierten Schild trägt, sehen wir in halber Vorderansicht. Er dreht den Kopf nach links und schaut gegen den Boden außerhalb des Bildes. Der andere, von einem weiten Mantel umhüllt, springt eilends fort. Der halbe Oberkörper und der Kopf werden vom I verdeckt. In der Mitte schwebt, fast mehr entspringend, ein Putto, den wir in halber Rückansicht sehen. In der Rechten hält er eine Siegesfahne, welche den Aufbau in Form eines Dreiecks noch unterstreicht. Die Spitze weist gegen zwei Reihen stilisierter Cumuluswölkchen. Schon im Münchner Evangeliar Heinrichs II., einer der ältesten abendländischen Darstellungen der Resurrectio, trägt der Auferstandene ein Siegeskreuz. In einer Miniatur der Initiale E zur Epistel der Ostermesse im Orationale von St. Erentrud in München erscheint die Siegesfahne als Zeichen der Überwindung des Todes. Schrade (1932) weist auf die besondere Wichtigkeit der österlichen Auferstehung unter allen Darstellungen christlichen Glaubensgutes. Paulus sieht in der Auferstehung Christi das Fundament des Christentums. Er schreibt im ersten Korintherbrief 15/13-14: «Ist aber Christus nicht auferstanden, so ist nichtig unsere Predigt und nichtig euer Glaube.» Bei Augustinus lesen wir: «Nimmt man den Glauben an die Auferstehung der Toten, so fällt die ganze christliche Lehre zusammen.» Keines der vier Evangelien beschreibt die Auferstehung. Sie melden sie als Tatsache: Im leeren Grab liegen die Leinentücher. Entsprechend zeigen die frühchristlichen Darstellungen nur das Grab und den Engel, welcher das Geheimnis der Auferstehung verkündet. Die Osterspiele mit ihrem Wechselgesang zwischen den Frauen und dem Engel führen allmählich zum künstlerischen 
Versuch, das österliche Geschehen als solches darzustellen. Im Münchner Evangeliar Heinrichs II., welches aus der Malschule von Reichenau, 2. Jahrzehnt des 11. Jahrhunderts, stammt, ist die Komposition frontal. Mehr seitlich erscheint der Auferstandene im Orationale von St. Erentrud, das als eines der Hauptwerke der Schule von Salzburg gilt. Nur die obere Hälfte der Gestalt ragt aus dem Grabe hervor. Das Verlassen des Grabes gleicht eher einem Herausklettern, wie es bei der Auferstehung der Toten beim Jüngsten Gericht dargestellt wird.

In späteren Darstellungen stellt der Auferstandene den einen Fuß auf den Grabesrand «wie der Sieger auf den Nacken des Feindes - devicta morte heißt es in der Oration der Ostermesse» (Schrade, 1932). Andere Darstellungen zeigen ihn mit einem $\mathrm{Fu}$ außerhalb des Grabes, «sodaß nun auch der Weg des Auferstandenen vom Grabe fort in die Welt hinein angedeutet wird» (Schrade, 1932). Mehr und mehr versuchen die Künstler die Resurrection in den Raum der Welt hineinzustellen: Auferstehung als Rückkehr. Im 13.Jahrhundert wird die Darstellung körperlicher. Die primitive Frontalansicht wird aufgegeben. Mit Leonardo, Raffael und MichelANGElo bekommt die Auferstehung den Charakter eines Hervorbrechens aus der Gruft, wobei die alte Vorstellung des «Saltus», des Überwindens großer Räume, erneut zu ihrem Rechte kommt. Solche Darstellungen finden wir auch bei Trzian. Sie könnten van Calcar beeinflußt haben. Auf der rechten Seite der Initiale I ist die mittlere Gestalt deutlich die eines Entspringenden. Die halbe Rückansicht unterstreicht diesen Eindruck des Enteilens. Sie ist den Auferstehungsbildern nicht ganz fremd. Wir sehen sie auf dem Tafelbild des Meisters Francke aus dem Anfang des 15. Jahrhunderts, welches in der Kunsthalle von Hamburg hängt.

Wurde die Gestalt des Auferstandenen im Verlauf der Jahrhunderte immer menschlicher, so ist sie auf der Initiale I des 7. Buches der «Fabrica» ins Spielerische umgebogen und stellt eine letzte Verweltlichung der Auferstehung dar, welche aber immer noch Symbol des leiblichen Todes und des ewigen Lebens bleibt.

\section{Literatur}

Anson B. J., The initial letters of 1555 edition of Vesalius' Fabrica, Quart. Bull. Northwest. Univ. Med. School Chicago 19 (1945) 326-335.

- Anatomical tabulae and initial letters in Vesalius' Fabrica and imitative works, Surg. Gynec. Obstetrics 89 (1949) 97-120.

Chaigneau G., L'illustration anatomique dans l'œuvre d'André Vésale, Diss. med. Paris 1935. 
Faller A., Die Entwicklung der makroskopisch-anatomischen Präparierkunst von Galen bis zur Neuzeit, Acta Anat. Suppl. 7 (1948).

- Anatomie und Chirurgie im Spiegel der Initialen von Vesals «Fabrica», 1955, Verh. Schweiz. Naturf. Ges. 131. Vers. Luzern (1951) 188-189.

- As iniciais da II ${ }^{a}$ dieçâo (1555) da obra «De Humani Corporis Fabrica Libri septem» de Andreas Vesalius, Festschrift $I^{o}$ Congresso Brasileiro de Historia da Medicina 1951, Rio de Janeiro 1951.

Francis H.S., The woodcut initials of the Fabrica, Bull. Med. Libr. Ass. 31 (1943) 228-239.

Herrlinger R, Die Initialen in Vesals anatomischem Lehrbuch, Ärztliche Praxis (Bad Wörishofen) 2 (1950) Nr. 26, S. 1-11.

- Die Initialen in Vesals «Fabrica», Index zur Geschichte der Medizin, Naturwissenschaft und Technik 1 (1953) 1-11.

- Die Initialen in der «Fabrica» des Andreas Vesalius, Acta Med. Hist. Patavina 10 (1963/64) 97-117.

HolläNDer E., Die Medizin in der klassischen Malerei, Enke, Stuttgart 1903.

Johnson F., Decorative Initial Letters, Cresset Press, London 1931.

LAMBERT S. W., The initial letters of the anatomical treatise, De humani corporis fabrica, of Vesalius, in S. W.LAmbert, W. Wiegand und W.M. Ivins jr, Three Vesalian essays to accompany the Icones Anatomicae of 1934, 1-24, Macmillan, New York 1952.

Loetzke H. H., Die großen und mittelgroßen Initialen in Vesals Hauptwerk, Anat. Anz. 126 (1970) 480-499.

Metzger Ch., Les lettrines de «l'anatomie» d'André Vésale, Scènes de la vie des anatomistes et des Chirurgiens au XVI ${ }^{\mathrm{e}}$ siècle, Hippocrate 3 (1935) 825-834.

Monteiro H., As letras capitulares do tratado de anatomia de Vesalio «De humani corporis fabrica» (Basileia 1543). Arquivo Anat. Antropol. 22 (1943) 433-476.

- L'ancienne technique de préparation des os. A propos des lettrines de la «Fabrica» de Vésale, Acta Anat. 14 (1952) 358-364.

Ollerenshaw R., The decorated woodcut initials of Vesalius' «Fabrica», Med. Biol. Ill. (London) 2 (1952) 160-166.

Pfister A., Über Anfänge und erste Entwicklung des Druckes medizinischer Werke in Basel, in Festschrift für Dr. J. Brodbeck-Sandreuter, 335-364, Benno Schwabe, Basel 1942.

Rosenkranz K., Die Initialen in Vesals Anatomie, Ein Beitrag zur Geschichte der anatomischen Abbildung, Sudhoffs Arch. 30 (1937/38) 35-46.

Rотн M., Andreas Vesalius Bruxellensis, Reimer, Berlin 1892.

Schmutzer R., Die Initialen in Vesals Anatomie, Sudhoffs Arch. 31 (1938) 328-330.

Schrade H., Ikonographie der christlichen Kunst, die Sinngehalte und Gestaltungsformen I, Die Auferstehung Christi, de Gruyter, Berlin/Leipzig 1932.

Spencer W.G., Vesalius, His delineation of the framework of the human body in the «Fabrica» and «Epitome» Brit. J. Surg. 10 (1922/23) 382-402.

Van Driessche A., Bij de initialen in Vesalius' werk, Verh. Vlaam. Acad. Geneesk. Belg. 16 (1954) 255-271.

Wells L.H., Note on a historiated initial letter in the Fabrica of Vesalius, Med. Hist. 6 (1962) $287-288$.

Wolf-Heidegger G. und A.M. Cetto, Die anatomische Sektion in bildlicher Darstellung, Karger, Basel 1966. 